Research Article

\title{
Spectrum-Effect Relationships of Flavonoids in Glycyrrhiza uralensis Fisch.
}

\author{
Tingting Li, ${ }^{1,2}$ Shiyao Hua, ${ }^{1}$ Jiahua Ma, ${ }^{1}$ Lin Dong, ${ }^{1}$ Fang Xu $\mathbb{D}^{1},{ }^{1}$ and Xueyan Fu $\mathbb{D}^{1}$ \\ ${ }^{1}$ Ningxia Medical University, Yinchuan 750004, China \\ ${ }^{2}$ Key Laboratory of Hui Ethnic Medicine Modernization, Ministry of Education (Ningxia Medical University), \\ Yinchuan 750004, China \\ Correspondence should be addressed to Fang Xu; xufang@nxmu.edu.cn and Xueyan Fu; xueyanfu2661@163.com
}

Received 27 June 2020; Revised 19 August 2020; Accepted 24 October 2020; Published 3 December 2020

Academic Editor: Eduardo Dellacassa

Copyright (C) 2020 Tingting Li et al. This is an open access article distributed under the Creative Commons Attribution License, which permits unrestricted use, distribution, and reproduction in any medium, provided the original work is properly cited.

Glycyrrhiza uralensis Fisch. is used in large quantities in traditional Chinese medicine. It contains flavonoids, saponins, and polysaccharides, with flavonoids being the main active ingredients. In this study, flavonoids were isolated from the roots of Glycyrrhiza uralensis Fisch. grown in 21 areas in China by water extraction, alcohol precipitation, polyamide resin separation, and other methods. Fingerprints were established by high performance liquid chromatography (HPLC). There were 15 common peaks in the fingerprints by similarity evaluations of the chromatographic fingerprints. The spectrum-effect relationships between the HPLC fingerprints and pharmacological activities of flavonoids in G. uralensis Fisch., including the heat clearing, detoxifying effects, cough relief, and phlegm elimination effects, were assessed by gray relational analysis and partial least squares regression. After HPLC-quadrupole time-of-flight mass spectrometry and standard comparison, these five identified compounds (liquiritin apioside, neoisoliquiritin, licochalcone $\mathrm{A}$, licochalcone $\mathrm{B}$, and licochalcone $\mathrm{C}$ ) could be used to evaluate licorice quality with regard to its efficacy. This research provides a scientific basis for improving licorice quality and also establishes a model for modernization of traditional Chinese medicines.

\section{Introduction}

Glycyrrhizae Radix et Rhizoma, the dried root and rhizome of Glycyrrhiza uralensis Fisch., Glycyrrhiza inflata Bat, or Glycyrrhiza glabra L. is commonly used in traditional Chinese medicine (TCM) [1] and described in the 2015 edition of the Chinese Pharmacopoeia. Glycyrrhiza uralensis Fisch. is one of the species included in the Pharmacopoeia [2]. It has a wide distribution and is planted over large areas in Northeast and Northwest China [3]. Licorice was first recorded in The Shennong Materia Medica and was described as having effects of invigorating the spleen and qi, clearing heat and detoxification, relieving coughing and eliminating phlegm, relieving spasms and pain, and mediating the effects of various medicines [3]. In modern pharmacological studies, many active components of licorice have been observed to have a range of pharmacological activities, including hypocholesterolemic, hypoglycemic, anxiolytic, antimicrobial, antiviral, free radical scavenging, antiulcer, cytotoxic, antitumor, antiallergic, antidiabetic, anticarcinogenic, antioxidant, anti-inflammatory, and hepatoprotective activities. Licorice is widely used in food, medicines, the chemical industry, and other fields $[4,5]$. At present, traditional formulations of licorice from other countries, such as Japan, Iran, and South Korea, are also widely used [6-8]. It is produced in China and is sold in many countries worldwide. Comprehensive development and utilization of licorice resources are expanding; then the market prospects are very broad. The content of total flavonoids and specific flavonoids in licorice from different sources varies greatly [6-8].

According to the 2015 edition of the Chinese pharmacopoeia, there are limitations to controlling glycyrrhizin and liquiritin. However, we do not know if their content can reflect their clinical utility and can be used to control the quality of licorice. Therefore, it is of great significance to 
establish a method to evaluate the complexity and integrity of licorice and improve quality standards. Current evaluation models lead to neglect of the interaction effects of multicomponent and multitarget TCMs and do not allow for optimum quality control of licorice. Hence, it is important to establish a method to evaluate complex samples such as licorice.

The spectrum-effect relationship is an effective method to evaluate the quality of TCMs [9-11]. Spectrum-effect relationship studies focus on correlations between fingerprint characteristics and pharmacodynamic data. The qualities of TCMs are evaluated using chemical components and biological activity, and the relative contributions of different components to the efficacy are determined. The results can be used to identify components that are most closely related to the pharmaceutical effect and accurately reflect the quality of a TCM.

In this study, the chromatographic fingerprints of 21 different producing areas of licorice were obtained using HPLC following existing quality control methods for crude TCMs. Common peaks were determined using similarity evaluation system software to create chromatographic fingerprints. The compounds for the selected common peaks were identified by HPLC-quadrupole time-of-flight (QTOF) mass spectrometry (MS) and comparison with standard samples. Data from experiments investigating the heat clearing and detoxification effects and cough relief and phlegm elimination effects were used to establish spectrumeffect relationships that were assessed using gray relational analysis (GRA) and partial least squares (PLS) regression. Flavonoid components of licorice could be used as indicators for quality control of licorice which were connected with the traditional effects identified. This research provides a method and scientific data for comprehensively improving licorice quality control.

\section{Experimental}

2.1. Reagents and Experimental Animals. Twenty-one production areas of dried licorice (Table 1) were collected from different growing areas in China, including Ningxia, Gansu, Inner Mongolia, Xinjiang, and Heilongjiang, in 2017, and identified by professor Zhang Xinhui at Ningxia Medical University (Yinchuan, China). Licorice standards (Table 2) were purchased from Yuanye Biotechnology Co. Ltd. (Shanghai, China). We used the following solvents in the experiments: acetonitrile (purity $=100 \%$, HPLC-grade; Thermo Fisher Scientific, Waltham, MA, USA); glacial acetic acid (purity $=100 \%$, HPLC grade; Kaixin Chemical Industry Co. Ltd, Tianjin, China); HPLC grade methanol, ethanol, and analytical grade ethanol (purity $=95 \%$, HPLC grade); and xylene and other reagents (Damao Chemical Reagent Production, Tianjin, China). Specific pathogen free-grade ICR male mice weighing $18-22 \mathrm{~g}$ were provided by the experimental animal center at Ningxia Medical University (animal certificate No. SCXK (Ning) 2015-0001). The mice were raised at room temperature $\left(22^{\circ} \mathrm{C} \pm 2^{\circ} \mathrm{C}\right)$ with a relative humidity of $50 \%-60 \%$ and natural light. For the experiments involving mice, they were randomly divided into a model group, blank group, and licorice treatment group with 10 mice in each group. Disease was induced in the model group but not the blank group, and both groups were given sodium carboxymethyl cellulose (CMC-Na) aqueous solution. Mice in the treatment group were continuously administered $150 \mathrm{mg} / \mathrm{kg}$ licorice total flavonoids for 1 week, and those in the blank and model groups were fed CMC-Na once per day. The blank group was not subjected to any experimental treatment.

\subsection{Instrumentation and Chromatography Condition}

2.2.1. Chromatography Condition. The chromatographic separation was performed on a ZORBAX SB-C ${ }_{18}$ Column $(250 \mathrm{~mm} \times 4.6 \mathrm{~mm}, 5 \mu \mathrm{m}$; Agilent Technologies) maintained at $30^{\circ} \mathrm{C}$ and using a diode array detector (DAD) with the detection wavelength set to $310 \mathrm{~nm}$. The mobile phase was a mixture of $0.2 \%$ glacial acetic acid (A) and acetonitrile (B) with a flow rate of $1.0 \mathrm{~mL} / \mathrm{min}$. The sample injection volume was $20 \mu \mathrm{L}$ and the sample was separated using the following optimized gradient elution: $0-15 \mathrm{~min}, 15 \% \mathrm{~B} ; 15-25 \mathrm{~min}$, $15-20 \% \mathrm{~B} ; 25-70 \mathrm{~min}, 20-50 \% \mathrm{~B} ; 70-90 \mathrm{~min}, 50-70 \% \mathrm{~B}$; and $90-95 \mathrm{~min}, 70-15 \%$ B. The results were analyzed using Agilent Chem Station.

MS measurements were performed with a 6545 Q-TOF instrument equipped with an electrospray ionization source (Dual AJS ESI+, Agilent Technologies). The drying gas temperature and source temperature were maintained at $350^{\circ} \mathrm{C}$ and $120^{\circ} \mathrm{C}$, respectively. The MS capillary voltage, cone voltage, and frag mentor voltage were fixed at $3500 \mathrm{~V}$, $30 \mathrm{~V}$, and $130 \mathrm{~V}$, respectively. The drying gas flow rate was $10 \mathrm{~L} / \mathrm{min}$ and the nebulizer pressure was $40 \mathrm{psi}$. The mass scan range was $50-1000 \mathrm{~m} / \mathrm{z}$.

2.2.2. Methodological Validation. One sample solution was analyzed six times to determine the precision. The RSD values of the peak area of each major chromatograph were all less than 3.5\%, and the RSD values of the relative retention time were all less than $1.8 \%$, indicating good precision of the instrument. A stability study was performed by analyzing a sample at different intervals over 1 day $(0,2,4,8,12$, and $24 \mathrm{~h}$ ). The RSD values of the peak-peak area of each major chromatograph were all less than $3.0 \%$, and the RSD values of the relative retention time were all less than $2.0 \%$, indicating that the sample solution was stable within $24 \mathrm{~h}$. Six sample solutions from the same batch were analyzed to determine the repeatability. The analysis of each sample was repeated three times. The RSD values of each main chromatographic peak area were all less than 5.0\%, and the RSD values of retention time were all less than $2.4 \%$, indicating that the method has good repeatability.

Then standard solution of the individual component was diluted gradually, to determine its limit of detection (LOD) values were determined by using signal-to-noise ratios of $10: 1$, as follows: liquiritin, $0.035 \mu \mathrm{g} / \mathrm{mL}$; isoliquiritin, $0.047 \mu \mathrm{g} / \mathrm{mL}$; liquiritigenin, $0.032 \mu \mathrm{g} / \mathrm{mL}$; isoliquiritigenin, $0.044 \mu \mathrm{g} / \mathrm{mL}$; liquiritin apioside, $0.025 \mu \mathrm{g} / \mathrm{mL}$; isoliquiritin apioside, $0.053 \mu \mathrm{g} / \mathrm{mL}$; neoliquiritin, $\quad 0.033 \mu \mathrm{g} / \mathrm{mL}$; neoisoliquiritin, $\quad 0.041 \mu \mathrm{g} / \mathrm{mL}$; 
TABLE 1: Glycyrrhiza uralensis Fisch. sample source information table.

\begin{tabular}{lcc}
\hline No. & Origin & Growth years \\
S1 & Weizhou, Tongxin County, Wuzhong City, Ningxia & Biennial \\
S2 & Lejing Township, Yanchi County, Wuzhong City, Ningxia & Biennial \\
S3 & Hooshawo Town, Yanchi County, Wuzhong City, Ningxia Hui Autonomous Region & Biennial \\
S4 & Zhaolou Village, Longde County, Guyuan City, Ningxia & Biennial \\
S5 & Shenlin Village, Longde County, Guyuan City, Ningxia & Biennial \\
S6 & Yuzhong County, Lanzhou City, Gansu Province & Biennial \\
S7 & Yongdeng County, Lanzhou City, Gansu Province & Biennial \\
S8 & Changning Town, Minqin County, Wuwei City, Gansu Province & Biennial \\
S9 & Qingshui Township, Min County, Dingxi City, Gansu Province & Biennial \\
S10 & Longxi County, Dingxi City, Gansu Province & Biennial \\
S11 & Xifeng District, Qingyang City, Gansu Province & Biennial \\
S12 & Huining County, Baiyin City, Gansu Province & Biennial \\
S13 & Songshan District, Chifeng City, Inner Mongolia & Biennial \\
S14 & Wanniute Banner, Chifeng City, Inner Mongolia & Biennial \\
S15 & Hangin Banner, Ordos City, Inner Mongolia & Biennial \\
S16 & Linxian County, Lvliang City, Shanxi Province & Biennial \\
S17 & Alar, Xinjiang & Biennial \\
S18 & Biennial \\
S19 & Horgos Port, Yili Kazakh Autonomous Prefecture, Xinjiang & Biennial \\
S20 & Xiangfang District, Harbin City, Heilongjiang Province & Biennial \\
S21 & Garsu & Billagennial \\
\hline
\end{tabular}

TABLe 2: The standards of licorice information table.

\begin{tabular}{lcc}
\hline Name of standards & CAS & Batch number \\
\hline Liquiritin & $551-15-5$ & Z10J8X39611 \\
Isoliquiritin & $5041-81-6$ & R07D8F50056 \\
Liquiritigenin & $578-86-9$ & Z20J8X40265 \\
Isoliquiritigenin & $961-29-5$ & R12A7F19390 \\
Liquiritin apioside & $74639-14-8$ & Y16D7Y306709 \\
Isoliquiritin apioside & $120926-46-7$ & P13A9F58700 \\
Neoliquiritin & $5088-75-5$ & Y18A7H19583 \\
Neoisoliquiritin & $7014-39-3$ & Y08J8H37579 \\
Glabridin & $59870-68-7$ & G006171216 \\
Licochalcone A & $58749-22-7$ & P21O8F46473 \\
Licochalcone B & $58749-23-8$ & P15D6F7553 \\
Licochalcone C & $144506-14-9$ & P13A9F58701 \\
Formononetin & $485-72-3$ & 16031005 \\
Glycyrrhizic acid & $1405-86-3$ & P11A9F58301 \\
\hline
\end{tabular}

glabridin, $\quad 0.058 \mu \mathrm{g} / \mathrm{mL}$; licochalcone A, $\quad 0.108 \mu \mathrm{g} / \mathrm{mL}$; licochalcone B $0.076 \mu \mathrm{g} / \mathrm{mL}$; licochalcone C, $0.012 \mu \mathrm{g} / \mathrm{mL}$; formononetin, $0.034 \mu \mathrm{g} / \mathrm{mL}$; glycyrrhizic acid, $0.056 \mu \mathrm{g} / \mathrm{mL}$; and $\beta$-glycyrrhetinic acid, $0.065 \mu \mathrm{g} / \mathrm{mL}$.

\subsection{The Total Flavonoids Content}

2.3.1. Preparation of Liquiritin Standard Solution. Liquiritin was precisely weighed and dissolved with $70 \%$ ethanol in a $10 \mathrm{~mL}$ volumetric flask to prepare a solution with a concentration of $1.05 \mathrm{mg} / \mathrm{mL}$.

2.3.2. Preparation of Sample Solutions. Samples of the licorice flavonoids concentrates were weighed to $30 \mathrm{mg}$ in $10 \mathrm{~mL}$ volumetric flasks and $70 \%$ ethanol was added. After ultrasonication for $30 \mathrm{mins}$, each sample was weighed and then stored at $4^{\circ} \mathrm{C}$ until required for analysis.
2.3.3. Standard Curve of Liquiritin. The linearity was studied by analyzing liquiritin standard solutions with five different concentrations and determining the absorbance by UV spectrophotometry at $213 \mathrm{~nm}$ (after full wavelength scanning, there is the maximum absorption wavelength). Taking the absorbance value $(A)$ as the $x$-axis, the concentrations of liquiritin were plotted on the $y$-axis to construct standard curves. The regression equation was $y=1.1299 x+3.2989$. The linear range was $0.021-0.105 \mathrm{mg} / \mathrm{mL}$ and the correlation coefficient was higher than 0.999 .

\subsubsection{Determination of Total Flavonoids of 21 Production} Areas. Preparation of sample solutions and method validation for quantitative analysis of total flavonoids content were stated in the supplemental material. The absorbance values for solutions of licorice total flavonoids from the 21 growing areas were determined using the method described in Section 2.3.3.

\subsection{HPLC Fingerprints}

2.4.1. Preparation of Sample Solutions. Preparation method was described in Section 2.3.2.

\subsubsection{Preparation of a Mixed Standard Solution.} Individual stock solutions at the following concentrations were prepared by dissolving standards in $70 \%$ ethanol: liquiritin, $4.07 \mu \mathrm{g} / \mathrm{mL}$; isoliquiritin, $4.07 \mu \mathrm{g} / \mathrm{mL}$; liquiritigenin, $3.38 \mu \mathrm{g} / \mathrm{mL}$; isoliquiritigenin, $4.15 \mu \mathrm{g} / \mathrm{mL}$; liquiritin apioside, $4.00 \mu \mathrm{g} / \mathrm{mL}$; isoliquiritin apioside, $3.84 \mu \mathrm{g} / \mathrm{mL}$; neoliquiritin, $3.69 \mu \mathrm{g} / \mathrm{mL}$; neoisoliquiritin, $3.08 \mu \mathrm{g} / \mathrm{mL}$; glabridin, $3.30 \mu \mathrm{g} / \mathrm{mL}$; licochalcone A, $3.54 \mu \mathrm{g} / \mathrm{mL}$; licochalcone B $3.08 \mu \mathrm{g} / \mathrm{mL}$; licochalcone C, $1.92 \mu \mathrm{g} / \mathrm{mL}$; formononetin, $3.69 \mu \mathrm{g} / \mathrm{mL}$; glycyrrhizic acid, $3.69 \mu \mathrm{g} / \mathrm{mL}$; and $\beta$-glycyrrhetinic acid, $3.69 \mu \mathrm{g} / \mathrm{mL}$. Take 100 
microliters of each standard solution and mix them together to make a mixed standard solution. After ultrasonication, the prepared solutions were stored at $4^{\circ} \mathrm{C}$ until required for use.

2.4.3. Similarity Evaluation of the Fingerprints. HPLC results for the samples from the 21 growing areas, including the retention times and peak areas, were exported in AIA (*.cdf) format in Chinese Medicine Fingerprint Similarity Evaluation (2004A Version). The median method is used to obtain the result. A representative reference fingerprint was automatically constructed using the median method by comparing the results for the 21 producing areas of licorice flavonoid extracts. Similarity values between the chromatogram for each batch and the reference fingerprint were calculated using software. Then, the results were used to identify common peaks for the licorice flavonoids.

2.4.4. Identification of Common Peaks. Under the conditions described in Section 2.4.2, the mixed standard and sample solutions were analyzed by HPLC-Q-TOF/MS. The spectrum of the mixed standard sample was compared with those of the sample solutions, and common peaks were identified according to the retention times and fragment ions. The flavonoid components for these peaks were inferred.

\subsection{Cough Relief and Phlegm Elimination Effects}

2.5.1. Phenol Red Excretion in Mice Trachea. One hour after the last dose, the mice were intraperitoneally injected with a $5 \%$ phenol red normal saline solution. After $30 \mathrm{~min}$, the mice were killed by dislocation. The trachea is separated and flushed with $0.5 \mathrm{~mL}$ of a $5 \%$ sodium bicarbonate $\left(\mathrm{NaHCO}_{3}\right)$ solution [9-11]. We combine the flushing solution into a small test tube and centrifuge at $2000 \mathrm{rpm}$ for $10 \mathrm{~min}$.

2.5.2. Ammonia-Induced Cough Test. Thirty minutes after the last treatment, the mice in the model and treatment groups were exposed to ammonia gas in a $500 \mathrm{~mL}$ beaker. The number of times the mice coughed in $2 \mathrm{~min}$ was recorded, with abdominal muscle contractions and yawning taken as coughing.

2.5.3. Sulfur Dioxide-Induced Cough Test. After the last dose, the mice were exposed to sulfur dioxide in a bell jar. For each group, the cough incubation period and the number of times the mice coughed within $3 \mathrm{~min}$ were observed and recorded [12].

\subsection{Heat Clearing and Detoxification Effects}

2.6.1. Mouse Acute Paw Swelling Tests. After the last dose, $0.1 \mathrm{~mL}$ of a $1 \%$ carrageenan solution was subcutaneously injected into the plantar area of each mouse [13]. The degree of swelling was calculated at $0,1,2,3,4$, and $5 \mathrm{~h}$ using the following equation: degree of swelling = foot volume at the measurement time - foot volume at $0 \mathrm{~h}$.

2.6.2. Mouse Acute Ear Swelling Test. After the last dose, the left ears of all the mice were uniformly coated with xylene, except for those in the blank group, which were coated with distilled water. The mice were euthanized $1 \mathrm{~h}$ after the ears were coated. The left and right ears of the mice were cut off, and pieces of the ear were taken from the same position with an $8 \mathrm{~mm}$ perforator and weighed, and the degree of swelling was calculated using the following equation: swelling = left ear mass - right ear mass.

2.7. Data Statistics. The experimental data are expressed as the means \pm SDs. The LSD and Dunnett's T3 test (3) were used for intergroup comparisons.

2.8. Statistical Analysis of the Spectrum-Effect Relationships. Correlation analysis was conducted between the common peak areas and pharmacodynamic data of licorice flavonoids from different regions using the HPLC data by GRA and PLS method. SPSS 24.0 (International Business Machines Corporation, New York, USA) and DPS 7.05 (Zhejiang University, Hangzhou, China) were used to process the data and find common peaks that were significantly related to the pharmacological effects.

\section{Results and Discussion}

3.1. Determination of the Total Flavonoids Content in Licorice. The total flavonoids contents in the 21 samples from different growing areas were calculated from the UV spectrophotometry results (Figure 1). The content of total flavonoids of licorice root was higher than 60\% except S16. The content of licorice flavonoids was the highest in S7. Licorice has a complex chemical composition [4, 14]. Flavonoids are one of the main active substances in licorice. Modern pharmacological studies have shown that it has multiple activities [15]. Studies have shown that the contents and types of licorice flavonoids from different sources vary greatly [16]. Licorice flavonoids are closely related to the traditional effects of licorice in TCM. In this study, the enrichment method can obtain relatively reliable licorice flavonoids and provide sufficient guarantee for further research.

3.2. Chromatographic Fingerprints and Similarity Analysis. The 21 licorice samples were injected into the HPLC and chromatograms were recorded. The results are shown in Figure 2. The spectrum of sample S1 was selected as the reference spectrum, and the software automatically matched the chromatographic peaks through multipoint correction. A control fingerprint (Figure 3) was generated by the selected median method, and 15 common peaks were analyzed. The similarity value of the generated control fingerprint (R) was set as one, and the similarities of the characteristic chromatograms of the 21 licorice samples were 


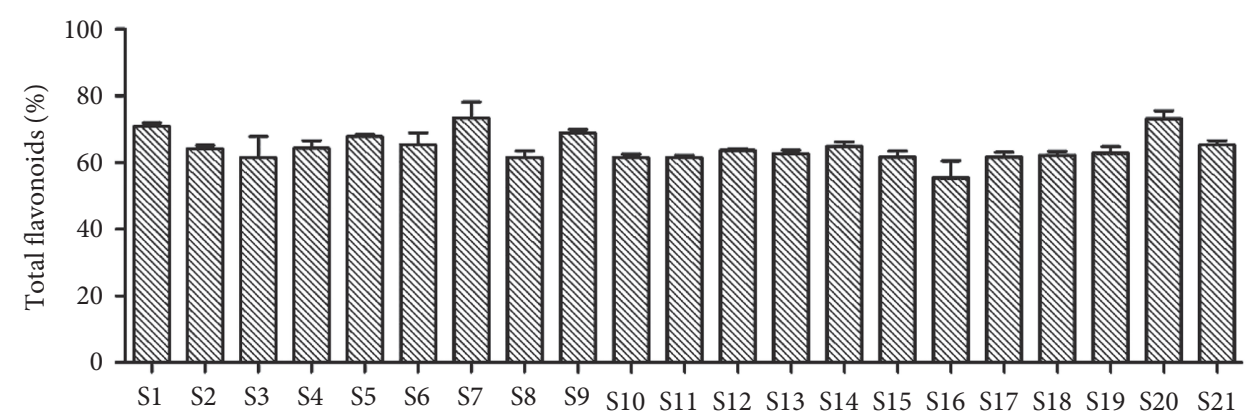

FIGURE 1: Determination of flavonoids in Glycyrrhiza uralensis Fisch. from 21 producing areas by UV. The result represents mean \pm S.D.

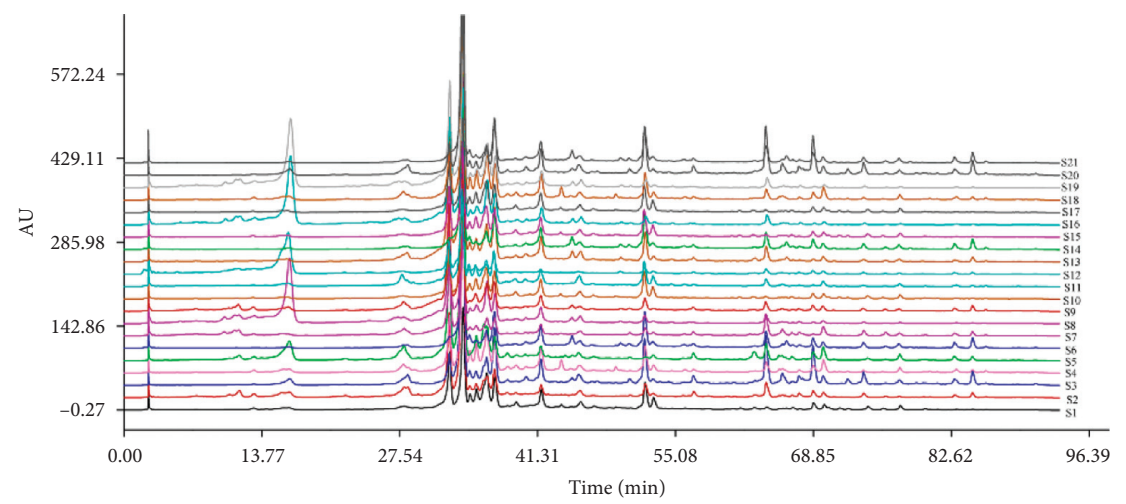

FIGURE 2: HPLC fingerprint of total flavonoids of Glycyrrhiza uralensis Fisch. from 21 producing areas.

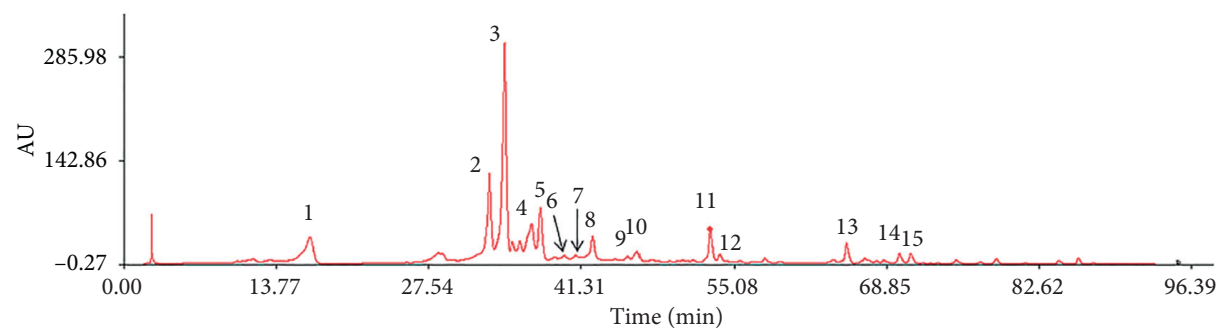

FIGURE 3: HPLC fingerprint of total flavonoids of Glycyrrhiza uralensis Fisch. from 21 producing areas.

calculated. The origins of the licorice samples and fingerprint similarities are shown in Table 3. Sixteen samples had similarities greater than 0.9 . We suspected that this difference between those samples could be caused by different producing areas of the licorice.

\subsection{Pharmacodynamic Experiments}

3.3.1. Test Results for Cough Relief and Phlegm Elimination. According to the tracheal phenol red experiment results (Figure 4), phenol red excretion in the model group was significantly reduced ( ${ }^{\# \#} P<0.01$ ) compared with the blank group, indicating successful modeling. Compared with the model group, the licorice flavonoids from samples S1-S21 significantly reduced the excretion of tracheal phenol red $\left({ }^{*} P<0.05\right.$ and $\left.{ }^{*}{ }^{*} P<0.01\right)$. The results of the ammonia cough test (Figure 5) showed that the total licorice flavonoids decreased the frequency of coughing in mice significantly compared with the model group, but to different degrees
( ${ }^{*} P<0.05$ and $\left.{ }^{*}{ }^{*} P<0.01\right)$. The licorice flavonoids had a therapeutic effect on coughing caused by ammonia. Samples S1, S2, S3, S8, S9, S10, S11, S12, S13, S17, and S18 significantly reduced coughing. For the sulfur dioxide cough test (Figure 6), compared with the model group, the licorice flavonoids reduced the frequency of coughing to different degrees $\left({ }^{*} P<0.05,{ }^{*}{ }^{*} P<0.01\right)$. Samples S1, S2, S3, S4, S8, S9, S10, S13, S14, and S18 showed the greatest reductions in coughing, whereas the antitussive effects of samples S5, S6, S7, S11, S12, S15, S16, S17, S19, S20, and S21 were slightly weaker.

\subsubsection{Test Results for Heat Clearing and Detoxification.} The results of carrageenan-induced mouse paw swelling tests (Figure 7) showed that paw swelling in mice treated with licorice samples S1, S2, S3, S5, S7, S8, S10, S13, S14, S16, S17, and $S 19$ was significantly $\left({ }^{*} P<0.05\right.$ and $\left.{ }^{*}{ }^{*} P<0.01\right)$ lower than that in the model group. The licorice flavonoids in the 
TABLE 3: Similarity analysis of fingerprint of total flavonoids of Glycyrrhiza uralensis Fisch. from 21 producing areas.

\begin{tabular}{lc}
\hline No. & Similarity \\
\hline S1 & 0.949 \\
S2 & 0.968 \\
S3 & 0.956 \\
S4 & 0.981 \\
S5 & 0.950 \\
S6 & 0.945 \\
S7 & 0.941 \\
S8 & 0.741 \\
S9 & 0.954 \\
S10 & 0.954 \\
S11 & 0.973 \\
S12 & 0.617 \\
S13 & 0.980 \\
S14 & 0.950 \\
S15 & 0.906 \\
S16 & 0.735 \\
S17 & 0.906 \\
S18 & 0.956 \\
S19 & 0.735 \\
S20 & 0.787 \\
S21
\end{tabular}

other samples did not significantly affect paw swelling in mice $(P>0.05)$. For the xylene-induced ear swelling in mice (Figure 8 ), the licorice flavonoids from all 21 samples significantly reduced ear swelling $\left({ }^{*} P<0.05\right.$ and $\left.{ }^{*}{ }^{*} P<0.01\right)$ compared with the model group.

\subsection{Spectrum-Effect Relationship Results}

3.4.1. Cough Relief and Phlegm Elimination Effects. With GRA Method, 14 peaks were identified with correlation degrees greater than 0.5 in the results of the tracheal phenol red experiment (Table 4). The order of the correlation degrees of these 14 peaks was $8>9>14>12>15>6>4>3>5>13>7>11>$ $10>2$. These peaks were closely related to the efficacy of licorice with regard to eliminating phlegm. From the ammonia exposure results (Table 5), 14 peaks were identified to have correlation degrees of greater than 0.5 with the frequency of coughing in mice. The contributions of these peaks were in the order $8>14>4>3>5>12>9>7>6>13>11>7>1>10>$ 15. These peaks were closely related to the efficacy of licorice with regard to coughing induced by ammonia. From the results of the sulfur dioxide cough tests in mice (Table 6), correlation degrees of 14 peaks were more than 0.5 . These peaks were in the order $15>9>14>12>5>4>8>3>11>7>6>10>13>1$. These peaks were closely related to the efficacy of licorice with regard to coughing induced by sulfur dioxide.

With the PLS regression method, the regression coefficients reflected the contribution of each $x$ to $y$. The larger the absolute value of the PLS regression coefficient, the greater the contribution of the peak to medical efficacy. Peaks were negatively correlated with the absorbance; that is, as the peak intensity increased, the absorbance value decreased. The remaining peaks were positively correlated with the absorbance. The pharmacodynamic experiment results of the tracheal phenol red test and the common peak areas were fitted by the regression equation $y=0.0348 \quad x 1-0.1386 \quad x 2+0.0808 \quad x 3+0.0890$ $x 4-0.1985 \quad x 5+0.0940 \quad x 6-0.0079 \quad x 7+0.0749 \quad x 8+0.1659$ $x 9-0.0857 x 10-0.1424 x 11+0.0952 x 12+0.0280 x 13+0.0136$ $x 14+0.0661 x 15$. In this regression equation, the contribution rates of the 15 common peak were in the order $5>9>11>$ $2>12>6>4>3>10>8>15>1>13>14>7$. For the ammonia cough test results, the regression equation from PLS regression analysis was $y=0.0055 x 1+0.0861 \quad x 2+0.0127$ $\begin{array}{lllll}x 3-0.0234 & x 4+0.0875 & x 5+0.0639 & x 6-0.0126 & x 7+0.0123\end{array}$ $x 8-0.0033 x 9+0.0170 \times 10+0.0540 x 11-0.0085 \times 12+0.0023$ $x 13+0.1469 x 14+0.1137 x 15$. The contribution rates of the 15 common peaks were in the order $14>15>5>2>6>11>$ $4>10>3>7>8>12>1>9>13$. The other peaks were positively correlated. For the mice sulfur dioxide coughing test results, the regression equation was $y=0.0054 x 1-0.0244$ $x 2-0.1618 \quad x 3-0.0506 \quad x 4+0.0741 \quad x 5-0.1443 \quad x 6+0.0460$ $x 7+0.0101 x 8+0.0269 x 9-0.0297 x 10+0.0608 x 11-0.0081$ $x 12-0.1336 x 13-0.1060 x 14-0.0805 x 15$. The contribution rates of the 15 common peaks were in the order $3>6>13>$ $14>15>5>11>1>4>7>10>9>2>8>12$.

Considering the results from the GRA and PLS methods, eight chromatographic peaks $(3,4,5,6,9,12,14$, and 15) were identified as being correlated with cough relief and phlegm elimination effects.

3.4.2. Heat Clearing and Detoxification Effects. With GRA method, the results of the carrageenan-induced paw swelling experiments in mice showed that 14 peaks had correlation degrees of greater than 0.5 with swelling (Table 7 ). The correlation degrees of these peaks were in the order $8>12>4>14>5>15>3>6>7>9>11>13>10>1$. These peaks were closely related to the efficacy of the drug with regard to the reduction of paw swelling. The results of the xyleneinduced ear swelling experiments in mice showed that 14 peaks had correlation degrees of greater than 0.5 with ear swelling (Table 8). The correlation degrees of these peaks were in the order $8>15>5>3>14>4>6>9>12>11>7>13>2>10$. These peaks were closely related to the efficacy of the drug with regards to the reduction of ear swelling.

With PLS regression method, the carrageenan-induced paw swelling results gave the regression equation $y=0.0017 x 1-0.0178$ $\begin{array}{lllll}x 2-0.1593 & x 3-0.0411 & x 4+0.0770 & x 5-0.0789 & x 6+0.0948\end{array}$ $x 7-0.0670 \quad x 8-0.0617 \quad x 9+0.0511 \quad x 10+0.0838 \quad x 11+0.0516$ $x 12-0.0423 x 13-0.0731 x 14-0.0558 x 15$. In this regression equation, the contribution rates of the 15 common peaks were in the order $3>7>11>6>5>14>8>9>15>12>10>13>4>2>1$. PLS regression of the xylene-induced ear swelling results gave the regression equation $y=-0.1294 x 1+0.0153 x 2+0.0687 x 3+0.0248 x 4+0.0140$ $\begin{array}{lllll}x 5+0.0827 & x 6+0.0346 & x 7+0.1777 \quad x 8+0.0692 & x 9+0.0648\end{array}$ $x 10+0.0646 x 11+0.0687 x 12+0.0886 x 13+0.0761 x 14+0.1347 x 15$. The contribution rates of the 15 common peaks were in the order $8>15>1>6>13>14>9>3>12>10>11>7>4>2>5$. Considering the results from the two methods comprehensively, seven chromatographic peaks $(3,5,6,7,8,14$, and 15) were identified as contributing to the heat clearing and detoxification effects. 


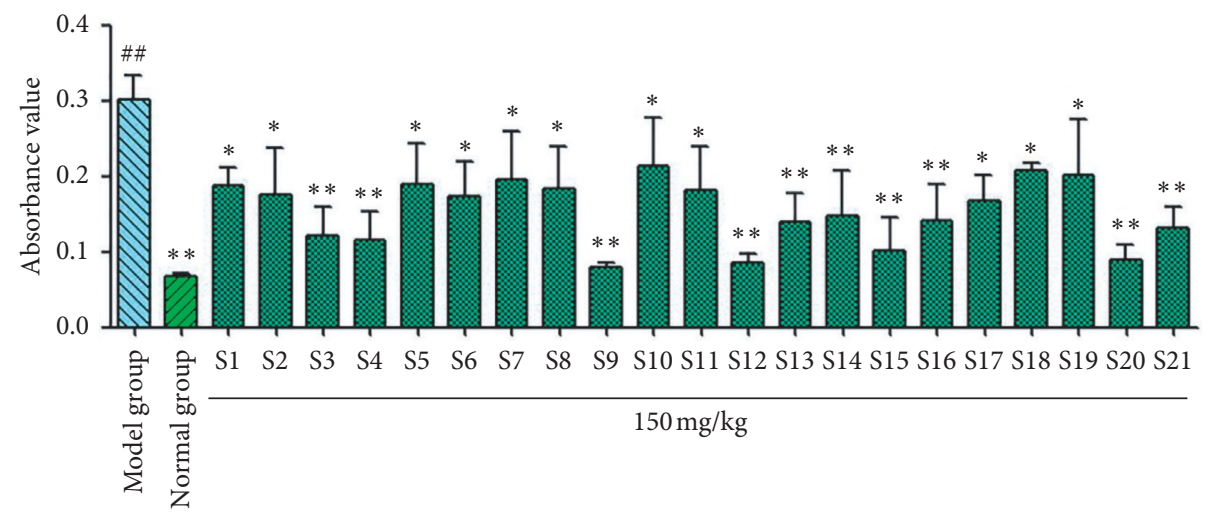

Figure 4: Results of tracheal phenol red of flavonoids in Glycyrrhiza uralensis Fisch. from 21 producing areas. The result represents mean \pm S.D. Note. Compared with the normal group, ${ }^{\# \#} P<0.01$ is a very significant difference. Compared with the model group, ${ }^{*} P<0.05$ was considered as significant difference; ${ }^{* *} P<0.01$, and there was a significant difference $(n=10)$.

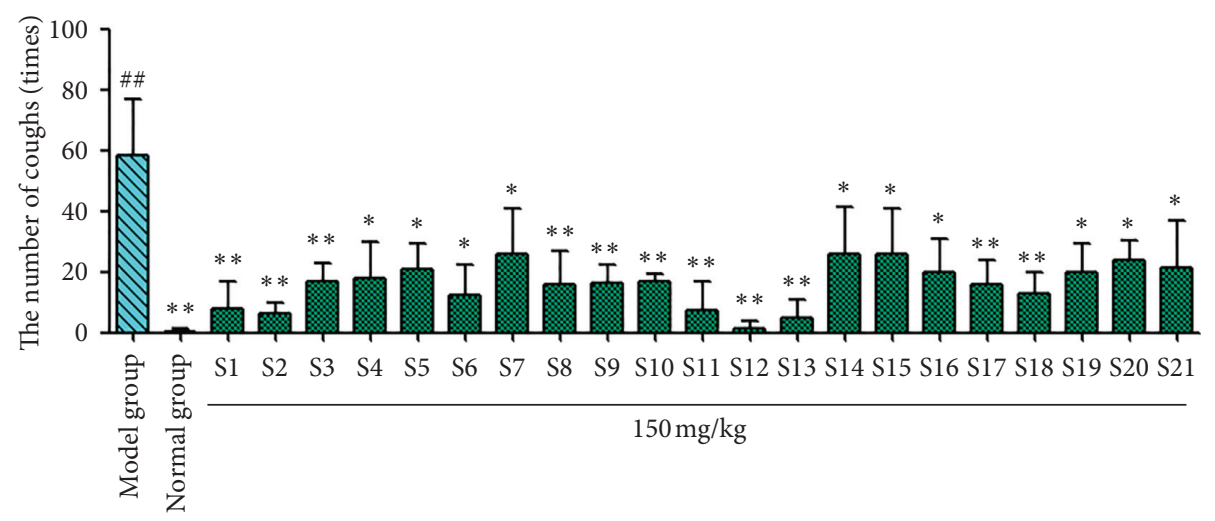

Figure 5: Results of ammonia-induced cough in Glycyrrhiza uralensis Fisch. flavonoids from 21 producing areas. The result represents mean \pm S.D. Note. Compared with the normal group, ${ }^{\#} P<0.01$ is a very significant difference. Compared with the model group, ${ }^{*} P<0.05$ was considered to be significant; ${ }^{*} P<0.01$, and there was a significant difference $(n=10)$.

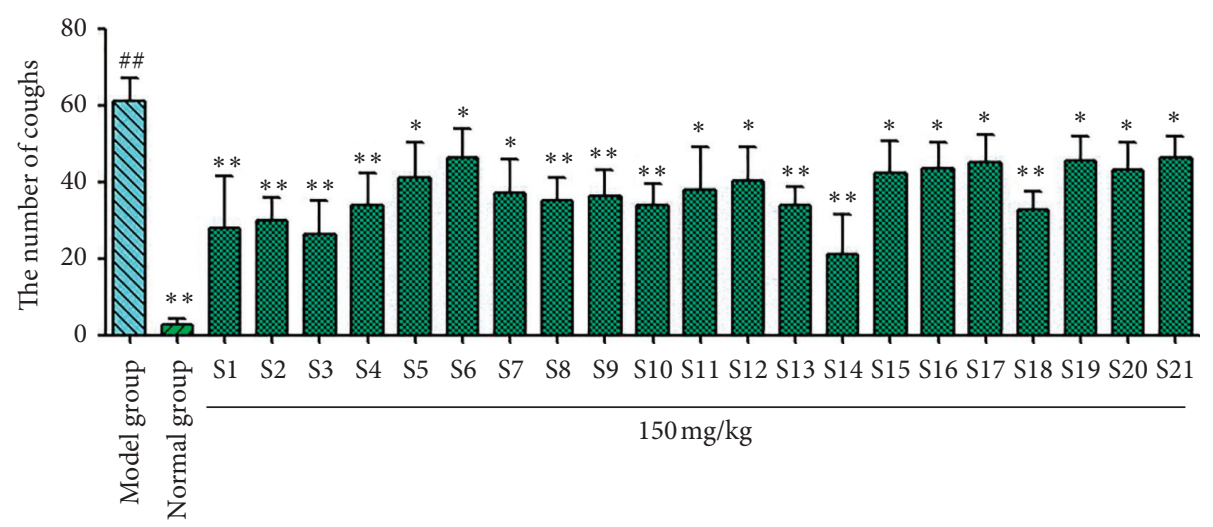

FIGURE 6: Results of $\mathrm{SO}_{2}$-induced cough in Glycyrrhiza uralensis Fisch. flavonoids from 21 producing areas. The result represents mean \pm S.D. Note. Compared with the normal group, ${ }^{\# \#} P<0.01$ is a very significant difference. Compared with the model group, ${ }^{*} P<0.05$ was considered to be significant; ${ }^{*} P<0.01$, and there was a significant difference $(n=10)$.

Licorice has attracted the attention of many researchers in recent decades. Many chromatographic techniques have been applied to licorice quality control $[1,17]$. Liu et al. used a single standard to quantify eight important active markers in licorice. The easily available glycyrrhizic acid was selected as a reference substance to calculate relative response factors [18]. These studies have improved the quality of licorice. Based on previous studies, we tried to use the Spectrumeffect relationship between HPLC fingerprints and effect to find the chemical components related to the efficacy and use 


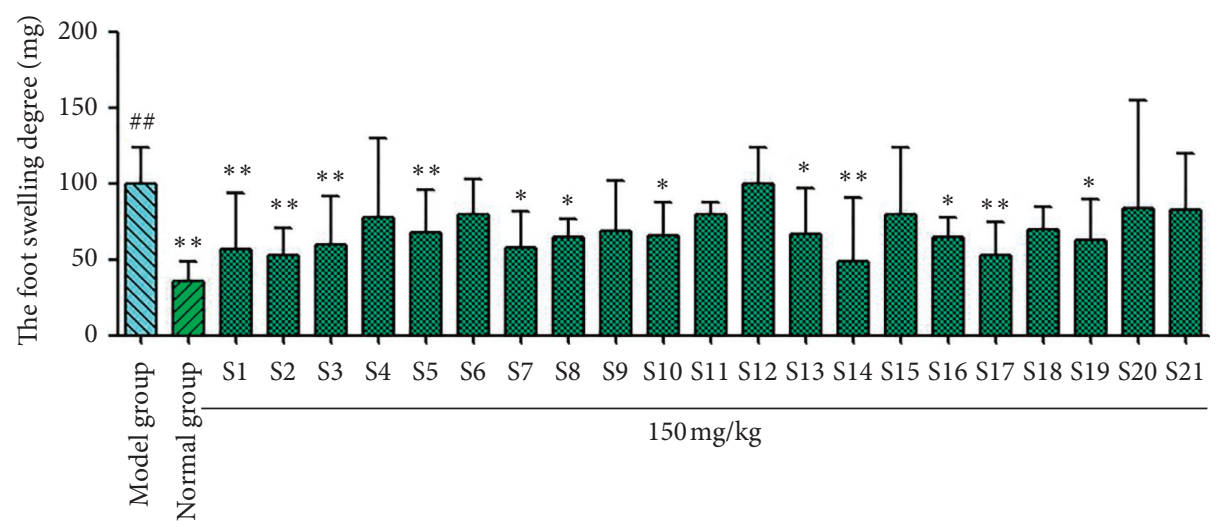

FIGURE 7: Results of foot swelling of Glycyrrhiza uralensis Fisch. flavonoids from 21 producing areas. The result represents mean \pm S.D. Note. Compared with the normal group, ${ }^{\# \#} P<0.01$ is a very significant difference. Compared with the model group, ${ }^{*} P<0.05$ was considered to be significant; ${ }^{*} P<0.01$, and there was a significant difference $(n=10)$.

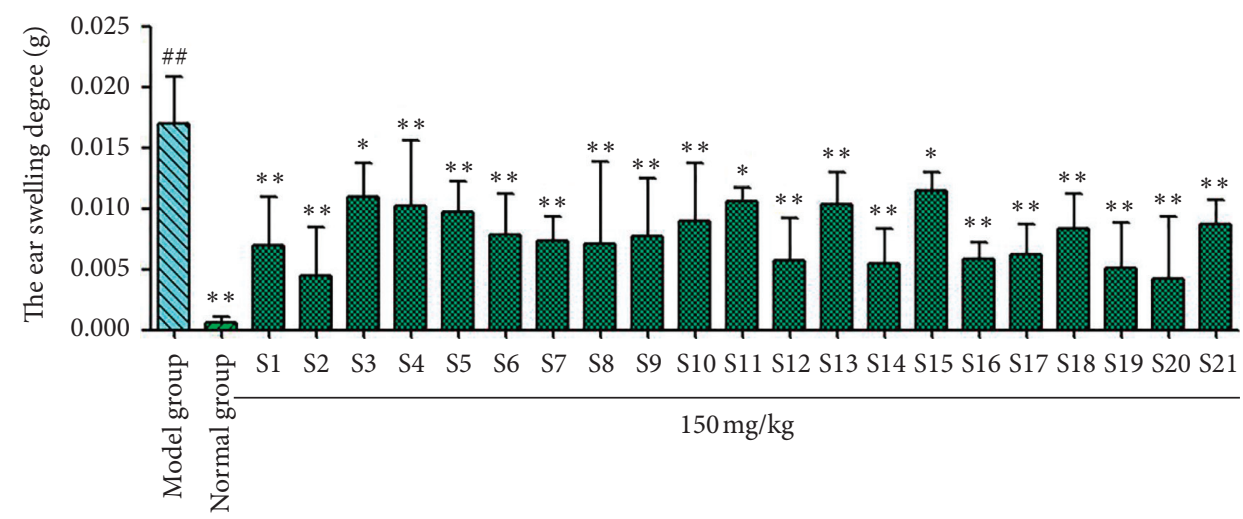

Figure 8: Results of ear swelling of Glycyrrhiza uralensis Fisch. flavonoids from 21 producing areas. The result represents mean \pm S.D. Note. Compared with the normal group, ${ }^{\# \#} P<0.01$ is a very significant difference. Compared with the model group, ${ }^{*} P<0.05$ was considered as significant; ${ }^{*} P<0.01$, and there was a significant difference $(n=10)$.

TABLE 4: Grey correlation between common peaks of flavonoids from Glycyrrhiza uralensis Fisch. and phenol red test in mouse trachea.

\begin{tabular}{lc}
\hline Peak number & Similarity \\
\hline 1 & 0.4529 \\
2 & 0.5355 \\
3 & 0.6313 \\
4 & 0.6361 \\
5 & 0.6170 \\
6 & 0.6383 \\
7 & 0.5820 \\
8 & 0.7090 \\
9 & 0.6676 \\
10 & 0.5455 \\
11 & 0.5814 \\
12 & 0.6494 \\
13 & 0.5977 \\
14 & 0.6504 \\
15 & 0.6419 \\
\hline
\end{tabular}

them as the quality evaluation criteria. The modern pharmacology research models selected in this study are representative of traditional models for evaluating efficacy. The main data analysis and processing methods currently
TABLE 5: Grey correlation between the common peaks of flavonoids from Glycyrrhiza uralensis Fisch. and ammonia-induced cough test.

\begin{tabular}{lc}
\hline Peak number & Similarity \\
\hline 1 & 0.5822 \\
2 & 0.4822 \\
3 & 0.6050 \\
4 & 0.6160 \\
5 & 0.6050 \\
6 & 0.5701 \\
7 & 0.5898 \\
8 & 0.6709 \\
9 & 0.5905 \\
10 & 0.5425 \\
11 & 0.5637 \\
12 & 0.5987 \\
13 & 0.5680 \\
15 & 0.6375 \\
\hline
\end{tabular}

available for spectrum-effect research are GRA and PLS regression. The GRA method can describe the size, strength, and order of factors using the gray correlation order. This can be used to determine the degree of influence of different 
TABLE 6: Grey Correlation between the common peaks of flavonoids from Glycyrrhiza uralensis Fisch. and $\mathrm{SO}_{2}$-induced cough test.

\begin{tabular}{lc}
\hline Peak number & Similarity \\
\hline 1 & 0.5338 \\
2 & 0.4392 \\
3 & 0.6082 \\
4 & 0.6344 \\
5 & 0.6389 \\
6 & 0.5801 \\
7 & 0.5903 \\
8 & 0.6251 \\
9 & 0.6521 \\
10 & 0.5776 \\
11 & 0.5923 \\
12 & 0.6457 \\
13 & 0.5704 \\
14 & 0.6493 \\
15 & 0.6572 \\
\hline
\end{tabular}

TABLE 7: Grey correlation between the common peaks of flavonoids from Glycyrrhiza uralensis Fisch. and foot swelling test.

\begin{tabular}{lc}
\hline Peak number & Similarity \\
\hline 1 & 0.5220 \\
2 & 0.4196 \\
3 & 0.6303 \\
4 & 0.6509 \\
5 & 0.6372 \\
6 & 0.6136 \\
7 & 0.6125 \\
8 & 0.7337 \\
9 & 0.6064 \\
10 & 0.5721 \\
11 & 0.5920 \\
12 & 0.6547 \\
13 & 0.5773 \\
14 & 0.6431 \\
15 & 0.6353 \\
\hline
\end{tabular}

TABLE 8: Grey correlation between the common peaks of flavonoids from Glycyrrhiza uralensis Fisch. and xylene-induced ear swelling in mice.

\begin{tabular}{lc}
\hline Peak number & Similarity \\
\hline 1 & 0.4001 \\
2 & 0.5457 \\
3 & 0.6575 \\
4 & 0.6488 \\
5 & 0.6957 \\
6 & 0.6404 \\
7 & 0.5959 \\
8 & 0.7537 \\
9 & 0.6366 \\
10 & 0.5245 \\
11 & 0.6179 \\
12 & 0.6249 \\
13 & 0.5887 \\
15 & 0.6548 \\
\end{tabular}

factors or the contributions of different factors to the main effects [19]. PLS regression is an effective method to study spectrum-effect relationships in TCMs $[13,20]$. The correlation degree can be evaluated using the correlation coefficient.

In this study, GRA and PLS regression analysis were applied to study the spectrum-effect relationships of the 21 licorice samples. The fingerprint and pharmacological data were analyzed to determine what peaks were closely related to the licorice efficacy. The important components of licorice were identified according to their contributions to the studied pharmacological effects. Finally, five flavonoids (peaks 3, 5, 6, 14, and 15) were identified as being closely related to the cough relief and phlegm elimination effects and heat clearing and detoxification effects.

3.5. Identification of Common Peaks. According to literature reports and expected cleavage of licorice compounds from PubChem and Sci Finder, a licorice database was established. Common peaks of the mixed standard solution and test solutions were subjected to HPLC-QTOF/MS in positive ion mode under the above conditions (Section 2.4.2). The analysis and matching were conducted by Agilent Mass Hunter Qualitative Analysis software using reference data, mass spectrometry data, and chromatographic retention data from the literature [21]. After this comparison, the following components were identified: liquiritin, isoliquiritin apioside, isoliquiritin, neoisoliquiritigenin, licochalcone $\mathrm{B}$, isoliquiritigenin, liquiritigenin, formononetin, licochalcone $\mathrm{C}$, and licochalcone $\mathrm{A}$. The five peaks $(3,5,6,14$, and 15) in the flavonoids fingerprint were closely related to its traditional efficacy, including liquiritin apioside, neoisoliquiritin, licochalcone $\mathrm{A}$, licochalcone $\mathrm{B}$, and licochalcone C (Table 9). 
TABLE 9: Assignment of common peaks in fingerprint of flavonoids in Glycyrrhiza uralensis Fisch. based on HPLC-Q-TOF/MS.

\begin{tabular}{lccccccc}
\hline Peak number & Name & Formula & RT & $\mathrm{m} / \mathrm{z}$ & Mass & Score & Theoretical isotopic \\
\hline 3 & Liquiritin apioside & $\mathrm{C}_{26} \mathrm{H}_{30} \mathrm{O}_{13}$ & 3.954 & 550.8892 & 550.5086 & 99.48 & $551.1700(24.42 \%)$ \\
5 & Neoisoliquiritin & $\mathrm{C}_{21} \mathrm{H}_{22} \mathrm{O}_{9}$ & 4.185 & 418.6043 & 417.1261 & 99.12 & $419.1272(20.87 \%)$ \\
6 & Licochalcone B & $\mathrm{C}_{16} \mathrm{H}_{14} \mathrm{O}_{5}$ & 4.745 & 286.1567 & 285.0841 & 98.12 & $287.0843(14.79 \%)$ \\
14 & Licochalcone C & $\mathrm{C}_{21} \mathrm{H}_{22} \mathrm{O}_{4}$ & 8.056 & 338.4099 & 337.3969 & 98.30 & $339.1501(20.21 \%)$ \\
15 & Licochalcone A & $\mathrm{C}_{21} \mathrm{H}_{22} \mathrm{O}_{4}$ & 8.332 & 338.4094 & 337.4039 & 98.63 & $339.1532(12.53 \%)$ \\
\hline
\end{tabular}

\section{Conclusions}

In this study, the HPLC spectrum-effect relationships of the licorice flavonoids for samples grown in 21 areas in China were studied by GRA and PLS regression. Five components of licorice have the largest contributions to the heat clearing and detoxification effects and phlegm elimination and cough relief effects of licorice. These components could be used for quality control of licorice. Our results provide a data basis for further identification of common peaks and a method for improvement of licorice quality control and drug efficacy evaluations.

\section{Data Availability}

The data used to support the findings of this study are included within the article.

\section{Conflicts of Interest}

The authors declare that they have no conflicts of interest.

\section{Authors' Contributions}

Tingting Li, Shiyao Hua, and Jiahua Ma contributed equally to this work.

\section{Acknowledgments}

The work was supported by the Ningxia Natural Science Foundation Project (Grant no. 2018AAC03097), the National Nature Science Foundation of China (Grant nos. 81860708 and 81560647), and the Ningxia Higher Education School First-class Discipline Construction Project (Chinese Medicine) Funded Project (Grant no. NXYLXK2017A06). The authors thank Gabrielle David, PhD, from Liwen Bianji, Edanz Group, China (http://www.liwenbianji.cn/ac), for editing the English text of a draft of this manuscript.

\section{Supplementary Materials}

Preparation of sample solutions and method validation for quantitative analysis of total flavonoids content. (Supplementary Materials)

\section{References}

[1] Y. Wang and Y. Yang, "Simultaneous quantification of flavonoids and triterpenoids in licorice using HPLC," Journal of Chromatography B, vol. 850, no. 1-2, pp. 392-399, 2007.

[2] China Medical Science, Pharmacopoeia of People's Republic of China, China Medical Science, Beijing, China, 2015.
[3] X. Li, L. Chen, and G. Li, "Ecological distribution and propagative technique research of Glycyrrhiza resources in China," Ecology and Environmental Sciences, vol. 22, no. 4, pp. 718-722, 2013.

[4] Q. Zhang and M. Ye, "Chemical analysis of the Chinese herbal medicine Gan-Cao (licorice)," Journal of Chromatography A, vol. 1216, no. 11, pp. 1954-1969, 2009.

[5] N. A. Mamedov and D. Egamberdieva, "Phytochemical constituents and pharmacological effects of licorice: a review," Plant and Human Health, vol. 3, pp. 1-21, 2019.

[6] M. Bahmani, M. Rafieian-Kopaei, S. A. Karamati et al., "A review of the health effects and uses of drugs of plant licorice (Glycyrrhiza glabra L.) in Iran," Asian Pacific Journal of Tropical Disease, vol. 4, pp. S764-S769, 2014.

[7] J. H. Lee, K. R. Ze, D.-H. Kim et al., "Analysis of liquiritigenin, an aglycone of liquiritin in licorice by high performance liquid chromatography," Korean Journal of Pharmacognosy, vol. 40, no. 4, pp. 309-314, 2009.

[8] S. M. Ju, M. S. Kim, Y. S. Jo et al., "Licorice and its active compound glycyrrhizic acid ameliorates cisplatin-induced nephrotoxicity through inactivation of p53 by scavenging ROS and overexpression of p21 in human renal proximal tubular epithelial cells," European Review for Medical and Pharmacological Sciences, vol. 21, no. 4, pp. 890-899, 2017.

[9] T. T. Zhu, L. Wu, X. L. Wang et al., "Investigation on relationships between chemical spectrum and bioeffect of prepared rhubarb decoction in rats by UPLC-ESI-Q-TOF-MS method coupled with gray correlation analysis," Journal of Functional Foods, vol. 31, pp. 104-112, 2017.

[10] W. Li, X. Sun, B. Liu, L. Zhang, Z. Fan, and Y. Ji, "Screening and identification of hepatotoxic component in evodia rutaecarpa based on spectrum-effect relationship and UPLCQ-TOFMS," Biomedical Chromatography, vol. 30, no. 12, pp. 1975-1983, 2016.

[11] Z. Shi, Z. Liu, C. Liu et al., "Spectrum-effect relationships between chemical fingerprints and antibacterial effects of Lonicerae japonicae flos and Lonicerae flos base on UPLC and microcalorimetry," Frontiers in Pharmacology, vol. 7, p. 12, 2016.

[12] S. P. Pattanayak and P. Sunita, "In vivo antitussive activity of Coccinia grandis against irritant aerosol and sulfur dioxideinduced cough model in rodents," Bangladesh Journal of Pharmacology, vol. 4, no. 2, pp. 84-87, 2009.

[13] Q. Chen, Research Methods in Pharmacology of Chinese Materia Medica, People's Medical Publishing House, Beijing, China, 3rd edition, 2011.

[14] T. Hatano, M. Takagi, H. Ito, and T. Yoshida, "Acylated flavonoid glycosides and accompanying phenolics from licorice," Phytochemistry, vol. 47, no. 2, pp. 287-293, 1998.

[15] S. Jia, "Research progress of the functions of licorice flavonoids," Journal of Beijing Union University, vol. 30, no. 4, pp. 67-73, 2016.

[16] G. Xing, N. Li, T. Wang, and M.-Y. Yao, "Advances in studies on flavonoids of Licorice," China Journal of Chinese Materia Medica, vol. 28, no. 7, pp. 593-597, 2003. 
[17] X. Qiao, C.-F. Liu, S. Ji, X.-H. Lin, D.-A. Guo, and M. Ye, "Simultaneous determination of five minor coumarins and flavonoids in Glycyrrhiza uralensis by solid-phase extraction and high-performance liquid chromatography/electrospray ionization tandem mass spectrometry," Planta Medica, vol. 80, no. 2-3, pp. 237-242, 2014.

[18] X. Liu, Q. Li, C. Lv et al., "Combination of the advantages of chromatographic methods based on active components for the quality evaluation of licorice," Journal of Separation Science, vol. 38, no. 24, pp. 4180-4186, 2015.

[19] S. Liu and Y. Lin, "Introduction to Grey systems modeling software," Understanding Complex Systems, vol. 68, pp. 287$302,2011$.

[20] X. Zhang, J. Chen, J.-L. Yang, and Y.-P. Shi, "UPLC-MS/MS analysis for antioxidant components of Lycii fructus based on spectrum-effect relationship," Talanta, vol. 180, pp. 389-395, 2017.

[21] Y. Zhao, S.-X. Liu, C.-X. Zhang, and D.-L. Liu, "Analysis on chemical constituents from Glycyrrhizae radix et Rhizoma by HPLC-Q-TOF-MS," Chinese Traditional \& Herbal Drugs, vol. 47, no. 12, pp. 2061-2068, 2016. 